\title{
48. Reading Information from a Sequential File (continued)
}

If a file contains more than one record, then the program has to open the file, process the records one-by-one, and finally close the file.

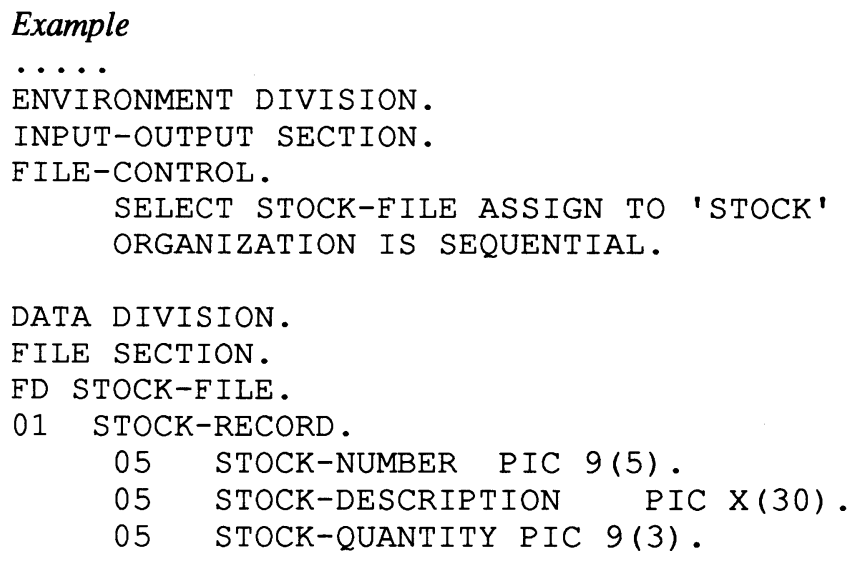

1. Write a program which will read the student file created by your program from lesson 46, exercise (1) and display the details.

2. Write a program which will read the staff file created by your program from lesson 46, exercise (2) and display the details. 